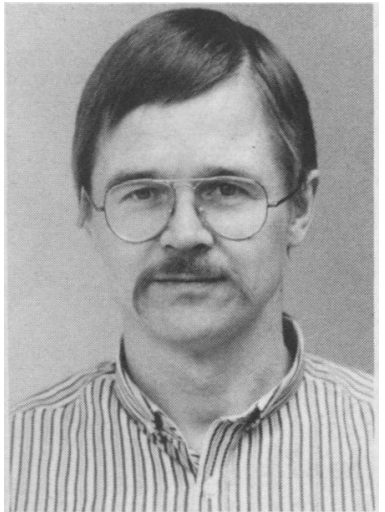

\title{
ANDROGENIC STEROID EFFECTS ON LIVER AND RED CELLS
}

\author{
M. ALÉN, MD \\ Dept. of Health Sciences, University of Jyväskylä, SF-40100 Jyväskylä, Finland, and \\ Research Unit for Sport and Physical Fitness, SF-40700 Jyväskylä, Finland
}

M. Alén

\section{ABSTRACT}

Haematological and hepatic effects of testosterone/anabolic steroid self-administration were investigated in five power athletes during 26 weeks of training.

During steroid administration blood haematocrit had increased $9.6 \%(p<.05)$ in the study group ( $n=5)$, but not in the control group $(n=6)$. This erythropoietic phenomenon was supported by increased $(p<.05)$ RBC and unchanged MCV. Blood haemoglobin concentration did not change markedly and consequently $\mathrm{MCHC}$ level in the study group decreased significantly ( $\mathrm{p}<.001$ ). Also the erythrocyte sedimentation rate decreased $(p<.05)$ in the study group.

The mean values of serum alanine aminotransferase, alkaline phosphatase and gamma-glutamyltransferase were and remained within normal range in both groups, although those of the study group were higher. The mean values of serum aspartate aminotransferase exceeded the normal range $(56 \mathrm{U} / \mathrm{l}$, at highest) but this may be of muscular rather than hepatic origin because of the severe training. It can be concluded that erythropoiesis was stimulated and liver function mildly impaired due to sustained highdose testosterone/anabolic steroid administration.

Key words: Testosterone, Anabolic steroids, Strength training, Liver function, Red cell count, Doping.

\section{INTRODUCTION}

The attempts to document objectively the effects and sideeffects of anabolic steroids have produced seemingly conflicting results (see e.g. Ryan, 1981; Lamb, 1984). As regards to haematological and hepatic phenomena, clinical studies have shown an increase in erythropoiesis (Gurney, 1976; Palacios et al, 1983) because of the administration of anabolic steroids, whereas most hepatological studies suggest that changes in serum transaminase, total bilirubin and alkaline phosphatase values during the use of androgens are both minor and infrequent (see e.g. Nishino, 1975). Hepatic lesions (peliosis and tumours) are, however, reported in patients treated with synthetic anabolic steroids (Turani et al, 1983; Overly et al, 1984). For other possible effects of such steroids on adult males the recent extensive review of Wilson and Griffin (1980) should be consulted.

Because it is not legal in Finland to get a physician's prescription for any drug to be used only for improving the performance of a healthy person, testosterone and/or anabolic steroids used as ergogenic aids are obviously obtained mainly from the black market. A recent trend in the use of these compounds seems to be the incorporation of testosterone among the anabolic steroids administered (Hill et al, 1983; Strauss et al, 1983). The simultaneous use of very high doses and several androgenic compounds leads to metabolic (Alén et al, 1985) and endocrine changes (Schürmeyer et al, 1984), and it can also be expected to result in haematological and hepatic changes.

The purpose of the present investigation was to study the

Correspondence to:

M. Alén, MD,

Department of Health Sciences,

University of Jyväskylä,

SF-40100 Jyväskylä, Finland effects of sustained high-dose use of testosterone and anabolic steroids in association with strength training on red blood cell values and liver function indicators.

\section{METHODS}

In the pilot study $\mathbf{4 0}$ male power athletes answered a questionnaire in which their habits of taking testosterone and anabolic steroids, and their future plans in training and hormone use were enquired. Altogether 15 of these men volunteered as subjects. Written informed consent was obtained from them all for the present study. The Ethical Committee of Kuopio University approved the study protocol.

Four subjects selected for the study were excluded because of profound change in the training programme and/or refusal to participate in all the tests performed.

The control group consisted of 6 athletes (3 powerlifters and 3 bodybuilders), who had decided not to take any steroid drugs during the next nine months of training and follow-up period. Two of them had earlier taken androgenic steroid (methandienone) for a few weeks but not during the 12 weeks preceding this study.

The study group comprised 5 athletes $(3$ bodybuilders, 1 powerlifter and 1 wrestler). They had not taken androgenic hormones during the 8-12 weeks preceding the study. All men in this group were experienced in the use of androgenic steroids. They had decided to selfadminister testosterone and anabolic steroids in a similar way to what they were previously used to during the following six months in combination with heavy resistance strength training. The self-administration of the androgenic steroids was followed by medication diaries and random urine analyse's with gas chromatography-mass spectrometry using the Varian MAT 212 instrument. On every occasion in every subject the random urine analyses were positive for the hormones listed in the personal drug diaries. Only one in this group took alcohol in small quantities, the others abstained.

The characteristics of the subjects studied are given in Table I, and Figure 1 summarises the time schedule of the study.

During the first 26 weeks of training (Phase 1) the study group self-administered anabolic steroids and testosterone (Fig. 2, Fig. 3 a-e). Methandienone (17a-methyl-17 $\beta$-hydroxy-1, 4-androstadien-3-one) was taken daily with slightly increasing doses. The intramuscularly selfinjected nandrolone (17 $\beta$-hydroxy-4-estren-3-one phenylpropionate) and stanozolol (17a-methyl-5a-androstano[3, 2-c] -pyrazol-17 $\beta$-ol) were 
TABLE I

Age, training background and anthropometric characteristics of the groups studied before and after 26 weeks of training. The values indicate mean + SEM. The intergroup values for significance levels are also given.

\begin{tabular}{|c|c|c|c|}
\hline Variable & $\begin{array}{l}\text { Study group } \\
\qquad(n=5)\end{array}$ & $\begin{array}{c}\text { Control group } \\
(n=6)\end{array}$ & t-test \\
\hline Age $(y r)$ & $27.0 \pm 2.5$ & $25.7 \pm 2.1$ & N.S. \\
\hline Strength training $(\mathrm{yr})$ & $7.4 \pm 2.6$ & $5.1 \pm 0.8$ & N.S. \\
\hline Height $(\mathrm{cm})$ & $177.8 \pm 1.5$ & $173.1 \pm 1.9$ & N.S. \\
\hline $\begin{array}{l}\text { Weight (kg) } \\
\text { Before }\end{array}$ & $86.8 \pm 5.1$ & $82.8 \pm 2.5$ & N.S. \\
\hline After & $92.0 \pm 4.1$ & $82.2 \pm 2.5$ & $p<0.05$ \\
\hline $\begin{array}{l}\text { Fat free weight }(\mathrm{kg}) \\
\text { Before }\end{array}$ & $72.8 \pm 3.4$ & $70.2 \pm 2.2$ & N.S. \\
\hline After & $80.6 \pm 3.3$ & $69.8 \pm 2.2$ & $p<0.05$ \\
\hline $\begin{array}{c}\text { Body fat (\%) } \\
\text { Before }\end{array}$ & $\begin{array}{l}15.6 \pm 2.9 \\
12.1 \pm 2.1\end{array}$ & $\begin{array}{l}15.3 \pm 2.2 \\
14.4 \pm 2.2\end{array}$ & $\begin{array}{l}\text { N.S. } \\
\text { N.S. }\end{array}$ \\
\hline
\end{tabular}

Phase I $\longrightarrow$ Phase II $\longrightarrow$

Strength training (with anabolic - steroids and testosterone in $\rightarrow$ Strength training
without steroids

Blood sampling, physical and medical examinations

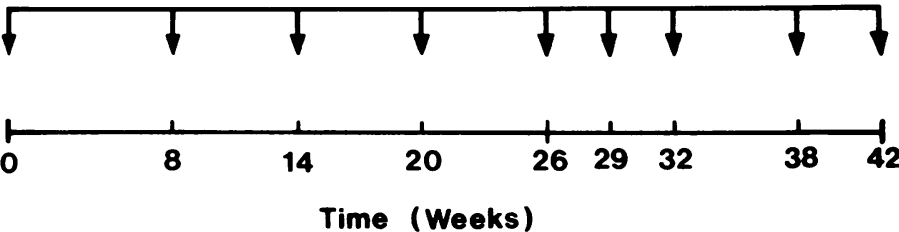

Fig. 1: Design of the investigation.

used usually weekly but the frequency of injections increased progressively, whereas the doses injected (both $50 \mathrm{mg}$ per injection) remained the same. Testosterone (17 $\beta$-hydroxy-4-androsten-3-one) was self-administered $(250 \mathrm{mg} /$ injection, consisting of testosteronepropionate $(30 \mathrm{mg})$-phenyl-propionate $(60 \mathrm{mg})$, -isocaproate $(60 \mathrm{mg})$ and -undecanoate $(100 \mathrm{mg}))$ initially twice a month but at the end of the study 4 times/month.

The study and the control groups had an intensive strength training programme during the investigation. Personal training diaries were filled in daily after each training session for subsequent control. Both groups trained for special purposes in their power event, and it included heavy resistance strength training, but no aerobic training for an average of six times per week. Because the subjects were highly motivated power athletes at top national level and had trained for competitive purposes for an average of 6.2 years, the exercises were only supervised randomly.

Random one week diet recalls, filled in daily, were completed three times by the subjects for the calculation of caloric intake and composition of the diet. The mean daily caloric intake in the study group was reported to be $15,400 \mathrm{~kJ}(3,700 \mathrm{Kcal})$ and in the control group $14,700 \mathrm{~kJ}(3,500 \mathrm{Kcal})$ and protein intake 2.3 and $2.6 \mathrm{~g}^{\times} \mathrm{kg}^{-1}$, respectively. This appears rather low for those athletes in heavy events involving strength training, but not for bodybuilders who avoided an increase in body fat.

After one day reduced training and overnight fast the subjects entered the laboratory at $\mathbf{8 . 0 0}$ a.m. for medical examinations and blood sampling. The height, weight, subscapular, triceps, biceps and crista iliaca skinfold thicknesses of the subjects were measured. The amount of body fat was estimated (see Table 1) according to Durnin and Rahaman (1967). The blood pressure was measured after ten minutes rest in the sitting position from the right upper arm with the sphygmomanometer.

Venous blood samples were drawn from the antecubital vein. Blood haemoglobin concentration ( $\mathrm{Hb}$ ) (analysed by the cyanomethaemoglobin method), haematocrit (PVC) (determined by microcentrifugation) and erythrocyte sedimentation rate (ESR) were analysed from EDTA blood and calculation of mean corpuscular haemoglobin concentration (MCHC) was also performed. The activities of serum aspartate amino-

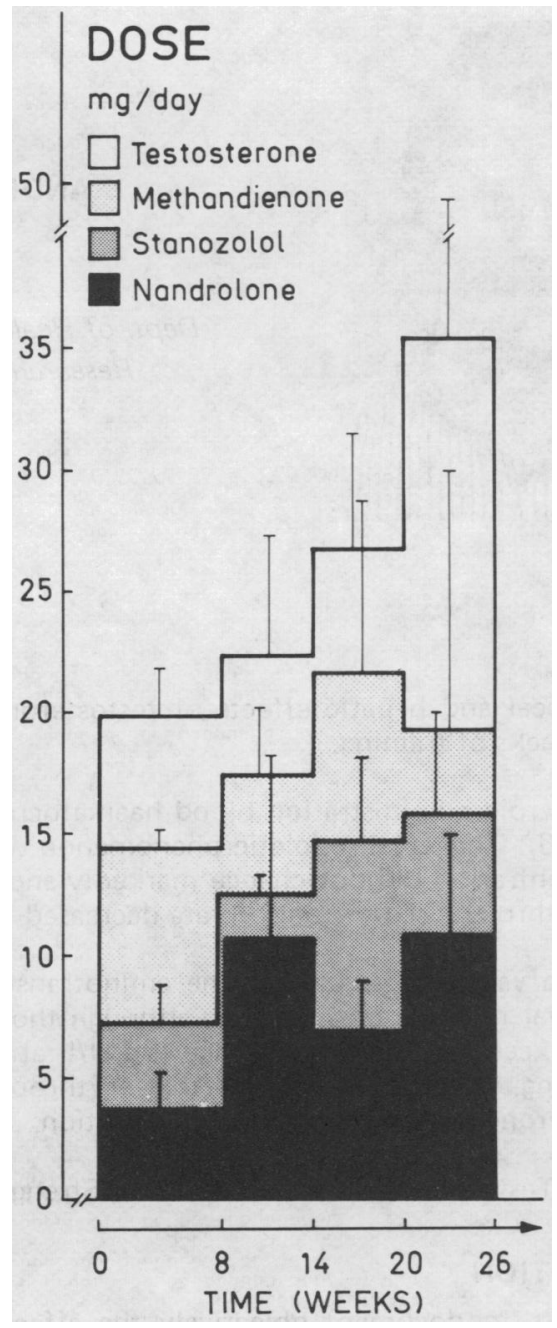

Fig. 2: Meal daily doses (+SEM) of self-administered testosterone and anabolic steroids ( $\mathrm{mg}$ ) in the study group.

transferase (ASAT), alanine aminotransferase (ALAT), gamma-glutamyl transferase $(\gamma-G T)$, alkaline phosphatase (AFOS) and total bilirubin concentration (TBIL) were analysed from serum samples stored at $-80^{\circ} \mathrm{C}$. The reagents of Boehringer Mannheim $\mathrm{GmbH}$ were used. These procedures were performed at $0,8,14,20,26,32,38$ and 42 weeks of training.

The following blood values were measured only before and after Phase 1: Red blood cell (RBC), platelet and leucocyte counting were carried out by Coulter Counter autoanalyser. Also the mean corpuscular volume (MCV) was calculated.

Statistical mothods. Means and standard errors were calculated. Differences between the mean values of the subject groups and between intragroup values were tested for significance by the two-tailed Student's t-test.

\section{RESULTS}

The mean values of $\mathrm{Hb}, \mathrm{PCV}, \mathrm{MCHC}$ and ESR are shown in Figure 4 a-d and those of ASAT, ALAT, AFOS and $\gamma-$ GT in Figure 5 a-d. Significant differences were not noticed between the study and control groups at the beginning of the investigation.

In blood $\mathrm{Hb}$ concentrations in the study group there was an initial decrease at 8 and 14 weeks (Fig. 4a), which then returned to pretreatment levels at 20 and 26 weeks. In general $\mathrm{Hb}$ values tend to be higher in the study group than in the control group.

PCV values had an increasing tendency in the study group throughout the period of testosterone/anabolic steroid administration (Fig. 4b) and the volume reached at 26 weeks $(51 \%)$ was significantly ( $p<.05$ ) higher than at the beginning of the investigation (46\%). Drug withdrawal led to a slow decrease in PCV and reached the pretreatment level at 38 weeks. Blood PCV was very stable in the control group.

MCHC values decreased significantly $(p<.001)$ in the study group during the first 14 weeks of steroid administration (Fig. $4 c$ ). Following 


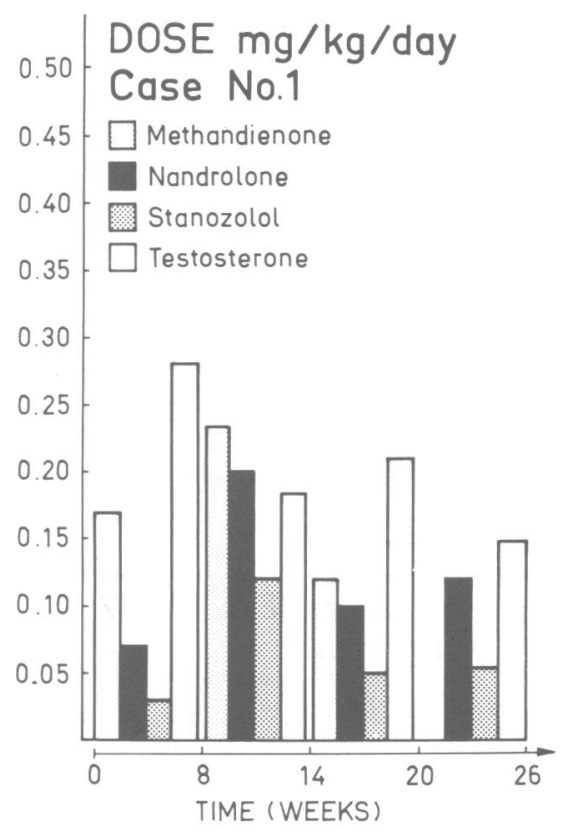

a

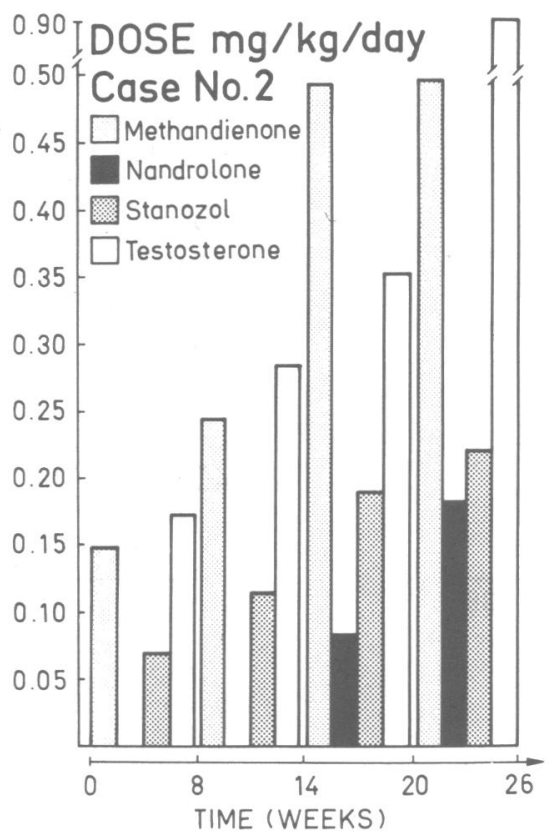

$b$

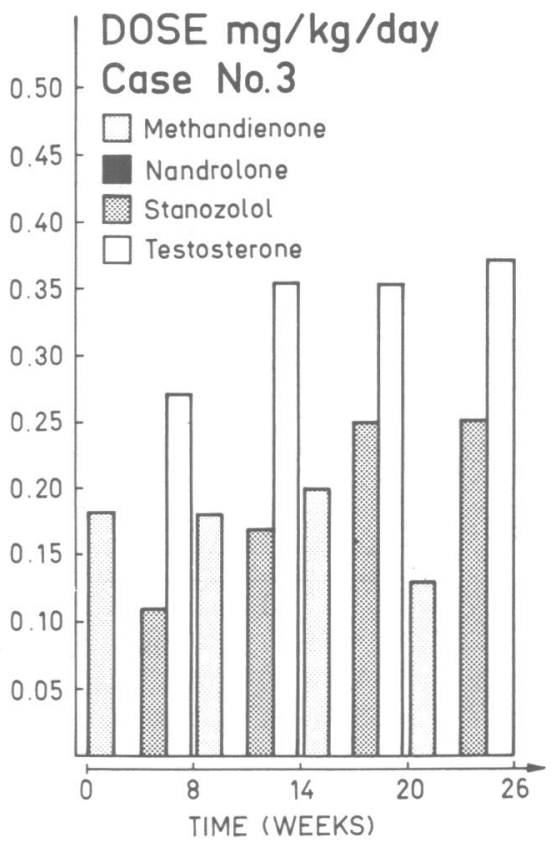

$C$

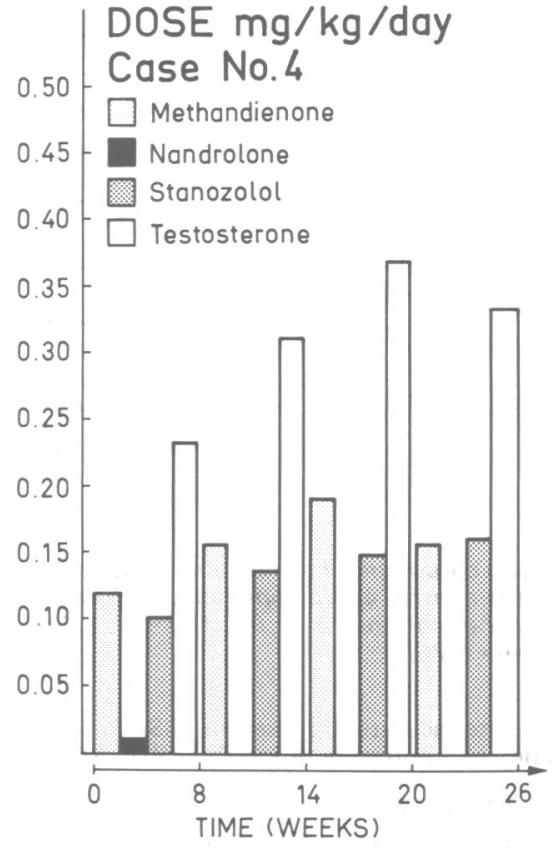

$d$

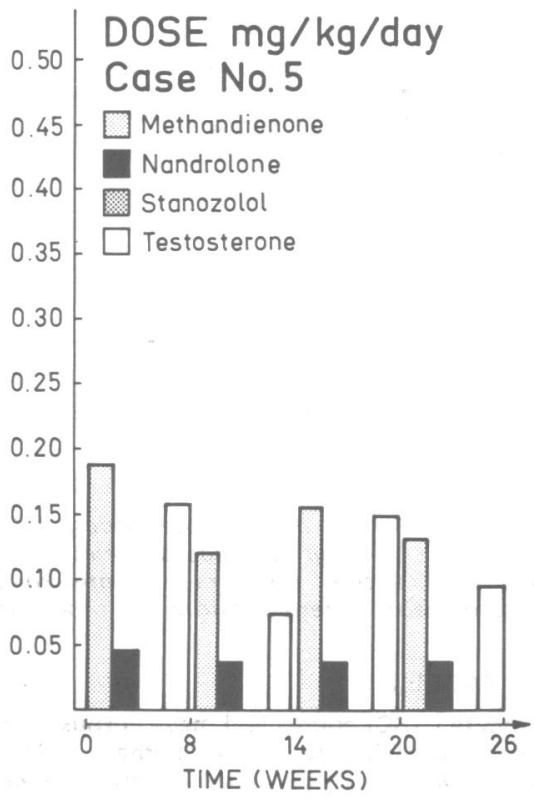

$e$

Fig. 3: Individual daily doses of testosterone and anabolic steroids (mg/kg). The values indicate means for the periods between the blood tests.

drug withdrawal MCHC returned relatively slowly to the onset level. There were some fluctuations in MCHC values in the control group but no clear-cut trends.

The values of blood ESR had a decreasing tendency in the study group during the androgenic steroid administration (Fig. 4d) and returned to the pretreatment level at 38 weeks. No systematic changes were observed in blood ESR in the control group.

The mean values of RBC, MCV, platelet and leucocyte count are given in Table II. Significant differences between the groups were not noticed before Phase 1 .

The mean value of RBC during Phase $I$ increased significantly ( $p<0.05$ ) in the study group, but did not change in the control group. Consequently the difference between the groups in RBC mean values was significant $(p<0.001)$ at 26 weeks.

No marked changes were noticed in MCV values in both group during Phase $\mathrm{I}$.
An increase in mean platelet count was observed in the study group but not in the control group. The difference between the groups was significant $(p<0.05)$ at 26 weeks.

Also the mean value of leucocyte count increased (p. $<0.05)$ in the study group by 26 weeks, while no change was observable in the control group.

$\mathrm{MCH}$ mean values also presented in Table II demonstrate difference ( $p<0.05$ ) between the groups at the end of Phase 1 .

The pattern of serum ASAT activities increased significantly $(p<.05)$ during the first 14 weeks of drug administration and remained at this higher level at $\mathbf{2 0}$ and $\mathbf{2 6}$ weeks (Fig. 5a). After drug withdrawal the values returned to the onset level in 12 weeks.

The activities of serum ALAT and AFOS were at higher level in the study group than in the control group. However, their mean values remained with in the normal range in both groups during the investigation (Figs. $5 b$ and $5 c$ ). 


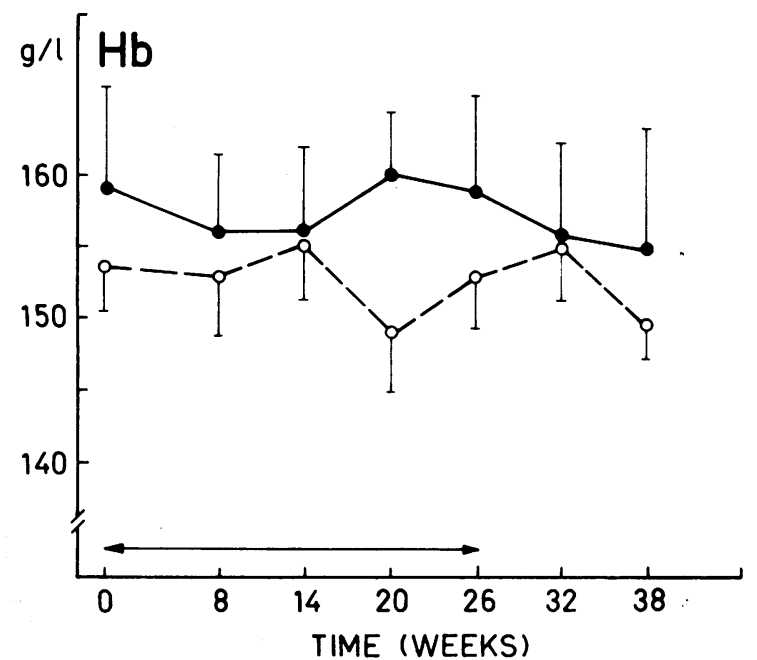

a

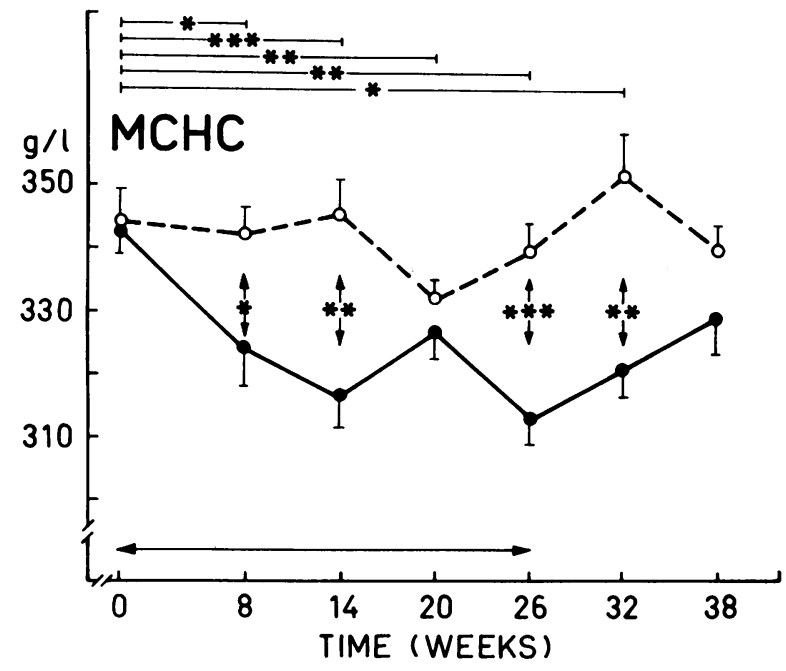

C

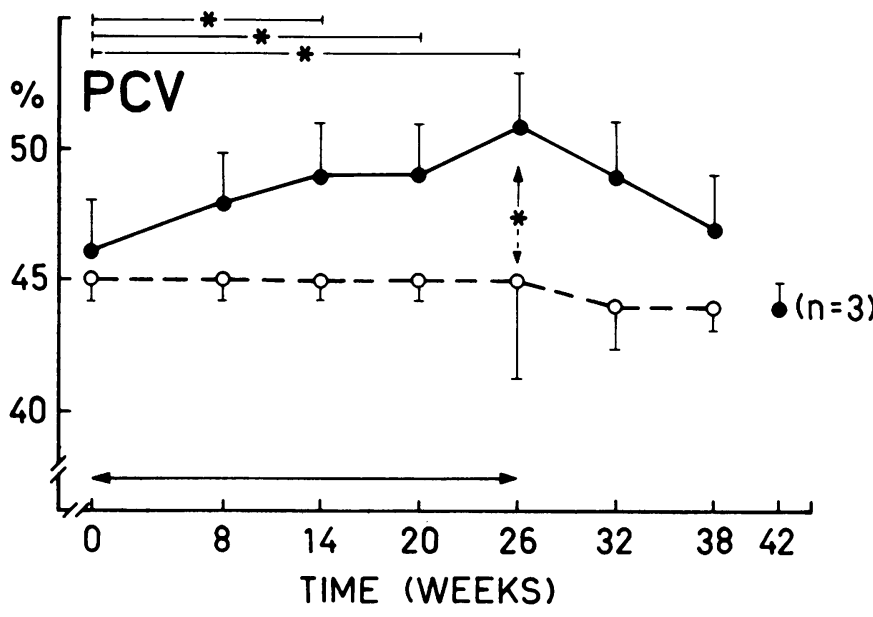

$b$

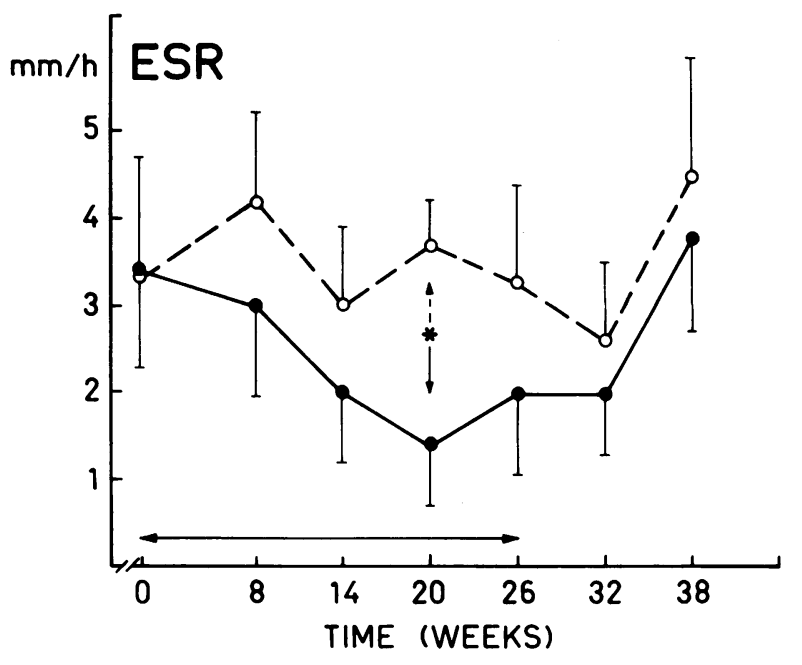

$d$

Fig. 4: The values of (a) blood haemoglobin (Hb), (b) hematocrit (PCV), (c) mean corpuscular haemoglobin concentration (MCHC), and (d) erythrocyte sedimentation rate (ESR) in the study group (closed circles, lines) and in the control group (open circles, broken lines). Mean values, standard errors of means and statistical significance of the intra- and intergroup differences are given ("p<.05, *"p<.01, ***p<.001). The study group self-administered testosterone and anabolic steroids during the time indicated (weeks 0-26).

\section{TABLE II}

Platelet, leucocyte and erythrocyte (RBC) counts and mean corpuscular volume (MCV) in the groups.studied before and after Phase I. The values indicate mean \pm SEM. The intergroup values for significance levels are also given.

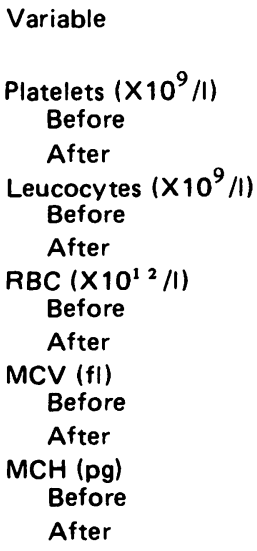

$$
\begin{array}{ccc}
\text { Control group } & \text { t-test } \\
(n=5) & (n=6) &
\end{array}
$$$$
316 \pm 33 \quad 281 \pm 23
$$$$
244 \pm 17
$$$$
6.3 \pm 0.3
$$$$
6.4 \pm 0.7
$$$$
6.1 \pm 0.5
$$$$
p<0.05
$$$$
7.4 \pm 0.4
$$$$
5.3 \pm 0.2
$$$$
5.1 \pm 0.2
$$$$
5.2 \pm 0.1
$$

N.S.

$p<0.05$

$$
5.8 \pm 0.3
$$

$89.6 \pm 0.6$

$88.8 \pm 2.2$

$89.8 \pm 0.7$

N.S.

p $<0.001$

N.S.

N.S.

N.S.

$30.5 \pm 0.9 \quad 30.6 \pm 0.3$

$30.5 \pm 0.3$

In serum $\gamma \cdot G T$ activities in the study group there was an initial significant ( $<<.05$ ) decrease at 14 weeks, which also remained at this lower level during the use of steroids. The return to pretreatment level took 12 weeks (Fig. 5 d).

The activities of serum ASAT, ALAT, AFOS and $\gamma-G T$ in the control group were relatively stable during the investigation.

There were no systematic changes from the initial concentrations of serum total bilirubin in the study and control groups (10.7 and $10.5 \mu \mathrm{mol} / \mathrm{l}$, respectively).

The values of blood pressure were initially at normal level ( $x$ 125/85 $\mathrm{mmHg}$ ) and no systematic changes were observed in any of the subjects studied.

\section{DISCUSSION}

The mean daily doses and the duration of administration of testosterone and anabolic steroids taken by athletes exceeded substantially those recommended for medical purposes and those administered in experimental conditions (Wilson and Griffin, 1980; Wright, 1980). Furthermore, a tendency to increase mean daily doses of exogenous androgens was observed and this was obviously due to decreased secretion of endogenous androgens (see e.g. Caminos-Torres et al, 1977; Schürmeyer et al, 1984). However, subject No. 5, who was less experienced in the use of testosterone and/or anabolic steroids took $25 \mathrm{mg} /$ day without any progression in doses or selfadministration frequency. His dose was only $38 \%$ of the total mean doses $(65 \pm 20 \mathrm{mg} /$ day) used by the four other athletes. Because of the main competition (of that year) subject No. 1 took androgens in progressive doses only until 14 weeks. 


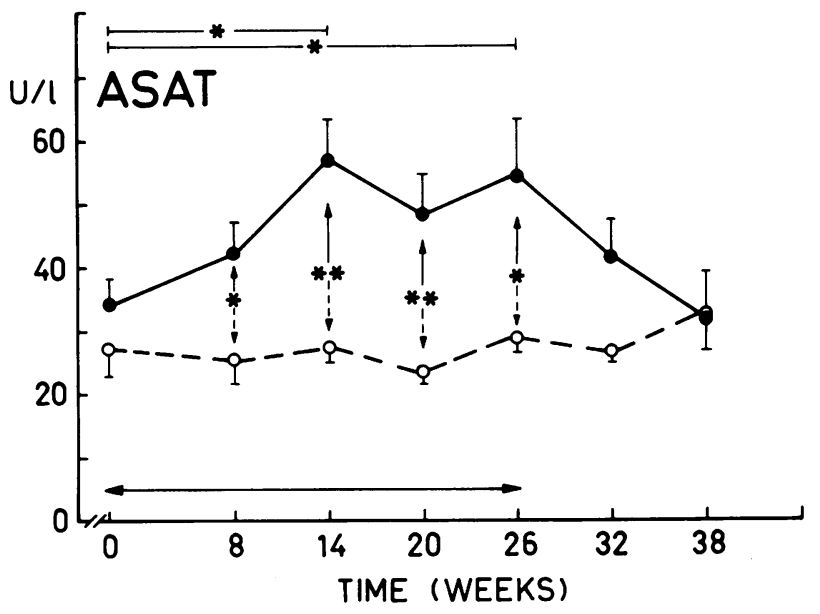

a

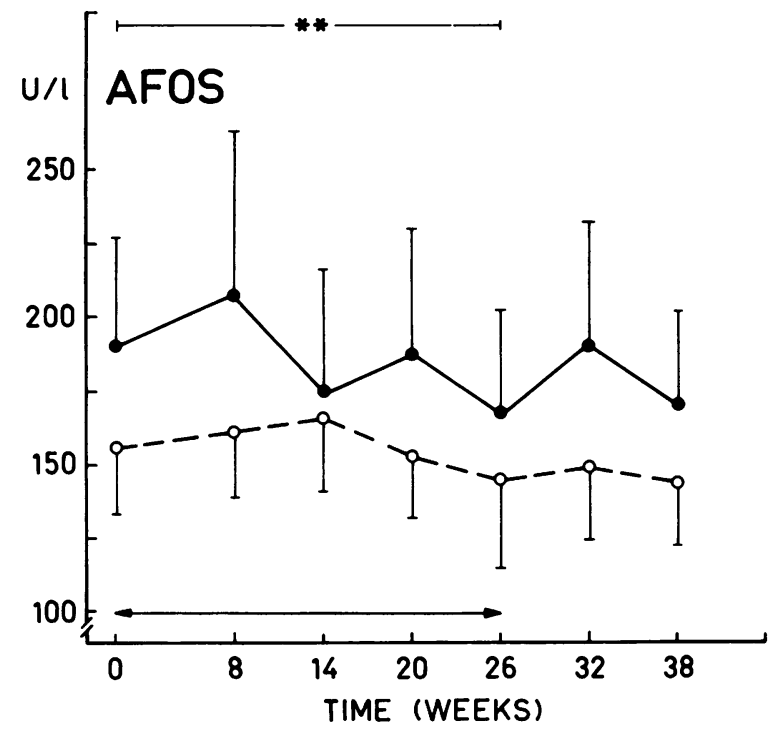

$c$

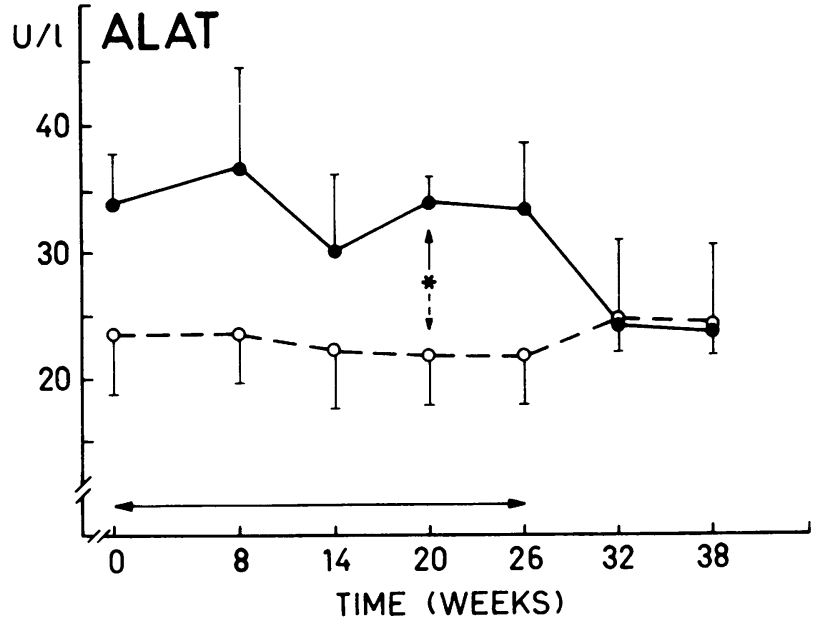

$b$

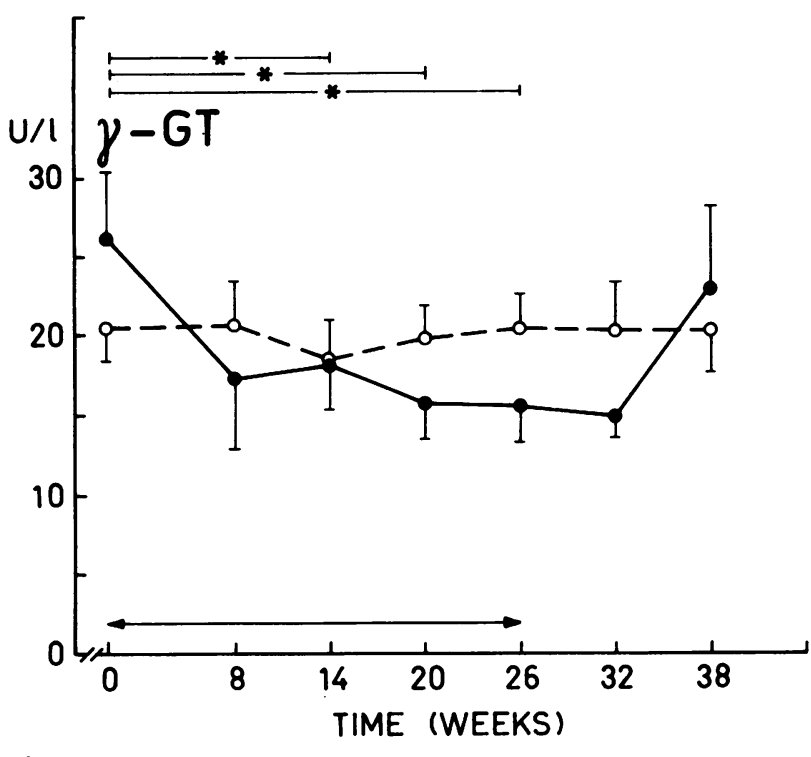

$d$

Fig. 5: Activities of serum (a) aspartate aminotransferase (ASAT), (b) alanine aminotransferase (ALAT), (c) alkaline phosphatase (AFOS), and (d) gamma-glutamyltransferase ( $\gamma-G T$ ) in the study group (closed circles) and in the control group (open circles). For other details see Fig. 4.

As expected this sustained high-dose use of testosterone and several anabolic steroids led to effects on haematological system and liver. There was a gradual increase in blood PCV in the study group to values not seen due to "pure" strength training. The mean of increase (in percentage) of PCV during Phase I was significantly higher in the study group as compared to that in the control group $(9.6 \pm 1.6$ vs. $1.1 \pm$ $1.7 ; p<.01)$. When the results of subject No. 5 were excluded from the analyses the difference between the groups in PCV mean values came out even more clearly. This erythropoietic phenomenon was supported by increased RBC and unchanged MCV values at the 26 week measurement. That was expected, since androgens and especially their $5 \beta$-metabolites stimulate erythropoiesis (see e.g. Necheles and Rai, 1969; Palacios et al, 1983).

As a consequence of elevated $\mathrm{PCV}$ and relatively stable $\mathrm{Hb}$ value the $\mathrm{MCHC}$ values decreased during the drug administra. tion. The mean decrease (in percentage) of MCHC was also steeper $(p<.01)$ in the study group $(8.8 \pm 1.8)$ than in the control group $(1.4 \pm 0.8)$. In association with the increase in PCV ESR mean values decreased in the study group.

As regards the liver it seems that 8 weeks was too short a time for recovery after drug withdrawal because serum ALAT was already in the beginning of the investigation at a higher level in the study group than in the control group. This higher level of serum ALAT remained relatively stable during the use of drugs but decreased statistically significantly (p $<.05$ ) within 12-16 weeks to the level of those controls after drug withdrawal. However, it is remarkable that the mean values in the study group were also within normal range although subject No. 5 was excluded. The function of the liver was obviously only slightly impaired because the activities of $\gamma$-GT in serum also remain within the normal range without any tendency to elevate. This finding was supported by the normal values of serum AFOS and TBIL.

The increase in serum ASAT levels in the study group may be of muscular rather than hepatic origin, since the athletes underwent severe and intensive strength training during the self-treatment (Tiidus and lanuzzo, 1983).

The stable level of serum ASAT in the control group is difficult to explain. Strength training was severe and intensive, since significant increases of $12.9 \%(p<.01)$ in the control group and $18.2 \%$ ( $p<.001$ ) in the study group took place in the maximal squat lift (Alén et al, 1984). It is suggested that training under the influence of exogenous androgens exposes the athletes to leaking of aspartate aminotransterase trom muscles to serum.

There also were some changes in the other blood and serum parameters measured in the control athletes. Because no clear- 
cut trend in those changes was observed they might have been random fluctuations within the normal range.

\section{ACKNOWLEDGEMENTS}

This study was financially supported in part by a grant from the Ministry of Education, Finland. The author would also like to thank Professor Eino Heikkinen, and Associate Professor Jorma Korvola and Mr. Paavo Rahkila, MSc for their cooperation.

\section{References}

Alén, M., Häkkinen, K. and Komi, P. V., 1984 "Changes in neuromuscular performance and muscle fibre characteristics of elite power athletes self-administering androgenic and anabolic steroids. Acta Physiol.Scand. 122: 535-544.

Alén, M., Rahkila, P. and Marniemi, J., 1985 "Serum lipids in power athletes self-administering testosterone and anabolic steroids. Int. J.Sports Med. (in press), 6 (2)

Caminos-Torres, R., Ma, L. and Snyder, P. J., 1977 "Testosteroneinduced inhibition of the $\mathrm{LH}$ and $\mathrm{FSH}$ responses to gonadotropinreleasing hormone occurs slowly". J.Clin.Endocrinol.Metab. 44: 1142-1153.

Durnin, J. and Rahaman, M., 1967 "The assessment of the amount of fat in the human body from the measurements of skinfold thickness". Brit.J.Nutr. 21: 681-689.

Gurney, C. W. (ed. Kochakian, C. D.), 1976 "The hematologic effects of androgens" in: Anabolic-Androgenic Steroids, pp. 483-497. Springer-Verlag, Berlin, Heidelberg, New York.

Hill, J. A., Suker, J. R., Sachs, K. and Brigham, C., 1983 "The athletic polydrug abuse phenomenon. A case study". Am.J.Sports Med. 11 (4): 269-271
Lamb, D. R., 1984 "Anabolic steroids in athletics: How well do they work and how dangerous are they?" Am.J.Sports Med. 12 (1): 31.38.

Necheles, T. F. and Rai, U. S., 1969 "Studies in control of haemoglobin synthesis: the in vitro stimulating effect of a $5 \beta-\mathrm{H}$ steroid metabolite on heme formation in human bone-marrow cells". Blood 34: 380-384

Nishino, Y. (ed. Laron, Z.), 1975 "Effects of androgens and related steroids on liver functions and enzymes" in: Pharmac.Therap.B. Vol. 1, No. 2, pp. 189-207. Pergamon Press, Great Britain

Overly, W. L., Dankoff, J. A., Wang, B. K. and Sing, U. D., 1984 "Androgens and hepatocellular carcinoma in an athlete". Ann.Int Med. 100 (1): 158-159.

Palacios, A Campfielf, L. A McClure, R. D., Steiner, B, and Swerdloff, R. S., 1983 "Effect of testosterone enanthate on hematopoiesis in normal men". Fertil.Steril. 40: 100-104.

Ryan, A. J., 1981 "Anabolic steroids are fool's gold". Federation Proc. 40: $2682-2688$.

Schürmeyer, T., Knuth, U. A., Belkien, L. and Nieschlag, E., 1984 "Reversible" azoospermia induced by the anabolic steroid 19 nortestosterone". The Lancet, 25 February, 1984, 417-420.

Strauss, R. H., Wright, J. E., Finerman, G. A. M. and Catlin, D. H. 1983 "Side effects of anabolic steroids in weight-trained men". The Physician and Sportsmed. 11 (12): 87-96.

Tiidus, P. M. and lanuzzo, C. D., 1983 "Effects of intensity and duration of muscular exercise on delayed soreness and serum enzym activities". Med.Sci.Sports Exerc. 15 (6): $461-465$.

Turani, H., Levi, J., Zevin, D. and Kessler, E. 1983 "Hepatic lesions in patients on anabolic androgenic therapy". Isr.J.Med.Sci. 19: 332 337.

Wilson, J. D. and Griffin, J. E., 1980 "The use and misuse of androgens". Metabolism 29 (12): 1278-1295.

Wright J. E. (ed. Hutton, R. S. and Miller, D. I.), 1980 "Anabolic steroids and athletics" in: Exercise and Sport Sciences Reviews, American College of Sports Medicine Series 8, pp. 149-198, Washington.

Title:

Authors: $\quad$ M. Jacob and P. Segal (Translated from French)

Publisher: $\quad$ Wolfe Medical Publications, London, 1984

Price: $£ 15$

I am not certain if this book has suffered from translation, or whether the original French version was equally complicated in its text layout. There is no index, but in its place, a list of abbreviations used throughout the book and their meanings. Every structure is given a leading letter abbreviation on first encounter and thereafter the abbreviation is used as the terminology, which at times leaves one swimming with a head full of letters and the inability to put the anatomy to them. The illustrations are simple and excellent, but again are ruined by the way in which structures are identified with abbreviations.

This is not the book for the uninitiated wishing to know more about knees, but it should have a place on the library of every knee surgeon as it does represent one school of thought in French literature. The book was designed by a team of doctors at the Rheims University Hospital Centre, with the main editors being Philippe Segal who studied with Albert Trillat at Lyon, and Marcel Jacob who is Physician to the French Soccer Federation.

The sections on rotary instability, both acute and chronic, are well worth going through carefully. If I have one further criticism it relates to the attitude taken in the book towards arthroscopy which seems to be far way behind the thinking in the rest of the world and is written off in a quarter of a page of text, less than that taken up by reference to the use of CAT scans in acute knee trauma.

J. Robertson

Title:

Editors: $\quad$ Bragg, Keats, Keffer et al 1984 YEAR BOOK OF RADIOLOGY

Publishers: $\quad$ Year Book Publications per Blackwells, Oxford

Price: $£ 37.00$

The Year Book of Radiology is an excellent digest of papers with reference to Radiology, taken from many journals. It is divided into sections; neuro-radiology, cardiac and interventional, thoracic, abdominal, paediatric radiology and radiation physics.

Only a small section of the book is of interest to those in sports medicine and orthopaedics. For instance, in this addition stress related fractures in the forearm are described in athletes and weight-lifters. There is a series describing stress fractures in the tarsal navicular which confirms the fact that we must look carefully at patients complaining of pain and also emphasises the use of radio isotopic bone scan as a screening procedure. There is a useful review of osteo-chondromatoses in athletes.

Although other papers are of use to radiologists and other clinicians, they are of little interest to readers of this journal. This is a book that belongs on the library shelves in which everyone should be prepared to read the parts related to their own interests and speciality. 